BMJ Open

Diabetes

Research

\& Care

\section{Interleukin-6 mediated exercise-induced alleviation of adiposity and hepatic steatosis in mice}

\author{
Long Li (D) , ${ }^{1,2}$ Caoxin Huang, ${ }^{1}$ Hongyan Yin, ${ }^{1}$ Xiaofang Zhang, ${ }^{1}$ Dongmei Wang, ${ }^{1}$ \\ Chen Ma, ${ }^{1}$ Jia Li, ${ }^{1}$ Yan Zhao, ${ }^{1}$ Xuejun Li (D) ${ }^{1}$
}

To cite: Li L, Huang C, Yin $\mathrm{H}$, et al. Interleukin-6 mediated exercise-induced alleviation of adiposity and hepatic steatosis in mice. BMJ Open Diab Res Care 2021;9:e001431. doi:10.1136/ bmjdrc-2020-001431

- Supplemental material is published online only. To view please visit the journal online (http://dx.doi.org/10.1136/ bmjdrc-2020-001431).

$\mathrm{LL}$ and $\mathrm{CH}$ contributed equally.

Received 2 April 2020 Revised 18 February 2021 Accepted 21 March 2021
Check for updates

(C) Author(s) (or their employer(s)) 2021. Re-use permitted under CC BY-NC. No commercial re-use. See rights and permissions. Published by BMJ.

${ }^{1}$ Department of Endocrinology and Diabetes, Xiamen Diabetes Institute, Fujian Key Laboratory of Translational Research for Diabetes, The First Affiliated Hospital of Xiamen University, Xiamen, Fujian, China ${ }^{2}$ Institute of Drug Discovery Technology, Ningbo University, Ningbo, China

Correspondence to

Dr Xuejun Li;

xmlixuejun@163.com and Dr

Yan Zhao;

zhaoyan1976@126.com

\section{ABSTRACT}

Introduction Exercise training has been shown to be the most effective strategy to combat obesity and nonalcoholic fatty liver disease. However, exercise promotes loss of adipose tissue mass and improves obesity-related hepatic steatosis through mechanisms that remain obscure.

Research design and methods To study the role of interleukin-6 (IL-6) in high-fat diet (HFD)-induced adiposity and hepatic steatosis during treadmill running, IL-6 knockout (IL-6 KO) mice and wild-type (WT) mice were randomly divided into lean, obese (fed a HFD) and trained obese groups (fed a HFD and exercise trained). Results After 20 weeks of HFD feeding and 8 weeks of treadmill running, we found that exercise obviously reduced HFD-induced body weight gain, inhibited visceral adipose tissue (VAT) and subcutaneous adipose tissue (SAT) expansion and almost completely reversed obesityrelated intrahepatic fat accumulation in WT mice. However, IL-6 knockout (IL-6 KO) mice are refractory to the benefits of treadmill training on body weight, VAT and SAT mass elevation, and hepatic steatosis. Moreover, a panel of lipolytic-related and thermogenic-related genes, including ATGL, HSL and PGC-1 $\alpha$, was upregulated in the VAT and SAT of WT mice that received exercise training compared with untrained mice, which was not observed in IL-6 K0 mice. In addition, exercise training resulted in a significant inhibition of hepatic peroxisome proliferator-activated receptor gamma (PPAR- $\gamma$ ) expression in WT mice, and these effects were not noted in IL-6 KO mice.

Conclusion These results revealed that IL-6 is involved in the prevention of obesity and hepatic fat accumulation during exercise training. The mechanisms underlying these antiobesity effects may be associated with enhanced lipolysis and thermogenesis in white adipose tissue. The improvement in hepatic steatosis by exercise training may benefit from the marked inhibition of PPAR- $\gamma$ expression by IL-6.

\section{INTRODUCTION}

Physical inactivity is linked to many chronic metabolic diseases, including obesity, type 2 diabetes, non-alcoholic fatty liver disease, sarcopenia and cardiovascular disease. In contrast, physical exercise is a powerful lifestyle intervention strategy to protect us against these metabolic dysfunctions by targeting

\section{Significance of this study}

What is already known about this subject?

- Exercise training has been shown to be the most effective strategy for many chronic metabolic diseases, including obesity, type 2 diabetes and nonalcoholic fatty liver disease.

- Few studies have investigated the mechanisms of interleukin-6 (IL-6) in high-fat diet (HFD)-induced adiposity and hepatic steatosis during exercise training.

What are the new findings?

- The antiobesity effects of exercise training are associated with IL-6-mediated pro-lipolysis and thermogenesis in both visceral and subcutaneous adipose tissue.

- The beneficial effects of exercise on obesity-related hepatic steatosis may be a result of IL-6 suppressing proliferator-activated receptor gamma (PPAR- $\gamma$ ) signaling.

How might these results change the focus of research or clinical practice?

- This study highlights IL-6 as an important mediator in the treadmill training-induced protective effects on adiposity and hepatic steatosis.

skeletal muscle, adipose tissues, liver and other organs by reducing visceral adiposity, enhancing insulin sensitivity, alleviating hepatic steatosis, preventing muscle atrophy and decreasing the risk of cardiovascular disease. ${ }^{12}$ The effects of exercise training on most metabolic diseases, especially adiposity and hepatic steatosis, are better than other available drugs so far. Either short-term or long-term exercise training could reduce both adiposity and obesity-related hepatic steatosis. ${ }^{34}$ However, how exercise improves these metabolic dysfunctions remains largely unknown.

Interleukin-6 (IL-6) is a pleiotropic cytokine produced by a variety of cell types with numerous functions in immunity, metabolism and tissue regeneration. Emerging data suggest a role of IL-6 in metabolic controls. 
IL-6-deficient mice developed mature-onset obesity with systemic insulin resistance. ${ }^{5}$ IL-6 injection in rodents enhanced glucagon-like peptide-1 production from pancreatic alpha cells and intestinal L cells and consequently improved insulin secretion and glycemia. ${ }^{6}$ Infusion of IL-6 in human adipose tissue stimulated lipolysis and fatty acid oxidation. ${ }^{7}$ Humanized anti-IL-6 receptor antibody treatment increased body weight and serum triglyceride (TG) and total cholesterol (TC) levels. ${ }^{8}$ Overexpression of the IL-6 gene reversed body weight gain and alleviated fatty liver and insulin resistance. ${ }^{9}$ Our previous study showed that administration of recombinant IL-6 protein protected against liver injury by promoting hepatic macrophage M2 polarization. ${ }^{10}$

An increasing number of cytokines known as myokines, which act as signal molecules in the cross talk between skeletal muscle and other organs to maintain the beneficial effects of physical activity, have been identified to be produced and released by muscle during exercise. ${ }^{11}$ Interestingly, IL- 6 has been identified as a myokine. Many different types of exercise have been reported to enhance its expression and secretion from contracting skeletal muscle in both rodents and humans. ${ }^{12}$ The IL-6 produced by skeletal muscle also contributes to energy metabolism and is necessary to elicit many reported benefits of exercise, including improved insulin sensitivity, reduced inflammation and increased fat oxidation. ${ }^{13} \mathrm{~A}$ recent randomized controlled clinical trial showed that tocilizumab (an anti-IL-6 receptor monoclonal antibody) could prevent bicycle exercise training-induced visceral fat loss. ${ }^{14}$ However, it is still unclear whether treadmill running plays the same role in regulating visceral adipose tissue (VAT) mass through IL-6 and whether IL-6 could mediate the effects of exercise on the accumulation of subcutaneous fat and intrahepatic triglycerides.

Herein, we hypothesized that exercise-elicited IL-6 potentially mediated the beneficial effects of exercise on adipose tissue and liver. In the present study, IL-6 KO mice and wild-type (WT) mice fed a high-fat diet (HFD) were used to determine whether exercise-induced visceral and subcutaneous fat loss was IL-6 dependent and to confirm whether IL-6 confers the beneficial effects of exercise on hepatic steatosis in mice. Furthermore, we investigated the molecular mechanisms regulated by IL- 6 that contribute to metabolic improvement by exercise training.

\section{RESEARCH DESIGN AND METHODS}

\section{Animal experiments}

C57BL/6 mice were obtained from Shanghai SLAC Laboratory Animal Co (Shanghai, China). IL-6 KO mice, which were generated on a C57BL/ 6 genetic background as previously described, were obtained from Jackson Laboratory (Bar Harbor, Maine, USA). IL-6 KO mice were backcrossed to C57BL/6 mice for at least 10 generations in our laboratory, and the mice for experiments were generated by heterozygote breeding, using corresponding WT littermates as controls. All procedures performed were approved by the Committee for Animal Research at Xiamen University and were in accordance with the guidelines for animal care and use. The mice were housed and maintained under controlled temperature $\left(21^{\circ} \mathrm{C}-23^{\circ} \mathrm{C}\right)$, humidity $(55 \%-60 \%)$ and lighting (12 hours light/dark cycles) and given water ad libitum. WT C57BL/ 6 mice and IL-6 KO mice were each randomly divided into three groups $(n=6-8 /$ group): (1) the control group received a normal diet; (2) the mice were fed a HFD (D12492, Research Diets) and kept sedentary; and (3) the mice were fed a HFD and received exercise training on a treadmill. Normal chow contained $70 \% \mathrm{kcal}$ from carbohydrates, $10 \% \mathrm{kcal}$ from fat and $20 \% \mathrm{kcal}$ from protein. High-fat chow contained $20 \%$ kcal from carbohydrates, $60 \%$ kcal from fat and $20 \% \mathrm{kcal}$ from protein. Forty-eighthours after the last exercise training, the mice were sacrificed with pentobarbital sodium anesthesia, and the tissues were harvested and quickly frozen in liquid nitrogen for biochemical and molecular analysis, or fixed in $10 \%$ neutral-buffered formalin for histological assays.

\section{Exercise training}

Mice were placed on the treadmill for $2 \mathrm{~min}$ before running; then, the treadmill speed was increased by $3 \mathrm{~m} /$ min every $2 \mathrm{~min}$ until $12 \mathrm{~m} / \mathrm{min}$. The mice kept running at $12 \mathrm{~m} / \mathrm{min}$ for $10 \mathrm{~min}$ on day $1,20 \mathrm{~min}$ on day 2 and 30 min on day 3 . After being acclimatized to the treadmill following the above protocol, the mice began regular treadmill running in a fixed program $(12 \mathrm{~m} / \mathrm{min}, 1$ hour per day, 5 days per week, 8 weeks). During the period that mice received exercise training, the mice in the HFD group remained sedentary in their cages. Food intake was detected during exercise training for a period of 21 days by measuring daily food changes.

\section{Hepatic TG measurement}

Liver tissues were homogenized in chloroform/methanol (2:1v/v) using a TissueLyser LT (QIAGEN, Shanghai, China). Lipid extracts were prepared by the classical Folch method. Extracts were dried under N2 flow and dissolved in isopropanol. TG contents were measured using a commercial triglyceride quantification colorimetric/fluorometric kit (BioVision, Wuhan, China) according to the manufacturer's instructions.

\section{Histological studies}

Fresh biopsy specimens from liver and adipose tissue were fixed in $10 \%$ neutral-buffered formalin for 24 hours, embedded in paraffin, sectioned using a Leica SM2010 $\mathrm{R}$ Sliding microtome (Shanghai, China) and stained with hematoxylin-eosin (HE) or Oil red $\mathrm{O}$ to assess histopathological features. For immunohistochemistry staining, adipose tissue sections were immunostained with anti-PGC-1 $\alpha$ (1:200, Abcam) antibody using a DAB Substrate Kit (MXB Biotechnologies, Fuzhou, China) and counterstained with hematoxylin. Stained areas were 
viewed and imaged using standard microscopy (Nikon, Shanghai, China).

\section{Plasma biochemistry and ELISAs}

For testing liver function, plasma alanine transaminase (ALT) concentrations were measured with commercial kits (Nanjing Jiancheng Bioengineering Institute, Nanjing, China) following the manufacturer's guidelines. The protein levels of plasma IL-6 were analyzed using an ELISA kit (R\&D Systems, Shanghai, China) according to the protocol provided by the manufacturer. All plasma biochemistry assays and ELISAs were determined by a Multiskan GO Microplate Spectrophotometer (Thermo Fisher Scientific, Shanghai, China)

\section{Western blot analysis}

Western blot analysis was conducted as previously reported. ${ }^{15}$ The target protein was detected using primary antibodies as follows: anti-proliferator-activated receptor gamma (PPAR- $\gamma$ ) (1:1000, Cell Signaling Technology), anti-PGC-1 $\alpha$ (1:1000, Abcam), anti-ATGL (1:1000, Cell Signaling Technology), anti-pHSL (1:1000, Cell Signaling Technology), anti-tHSL (1:1000, Cell Signaling Technology), anti- $\alpha$-tubulin (1:1000, Abcam) and anti- $\beta$-actin (1:2000, Cell Signaling Technology) .

\section{Real-time PCR}

Total RNA from liver tissues and adipose tissues was isolated using the RNA simple Total RNA Kit (Tiangen, Beijing, China) according to the manufacturer's instructions. cDNA was synthesized from total RNA using a FastQuant RT kit (Tiangen) according to the manufacturer's instructions. Quantification of mRNA was carried out on a Roche LightCycler 480 Real time PCR Machine using SYBR Premix Ex Taq II (Takara, Dalian, China). The quantitative values of mRNA were normalized relative to the levels of GAPDH.

\section{Statistical analysis}

Statistical analyses were carried out with GraphPad Prism V.8.0 software for Windows. All results are expressed as mean \pm SEM, with $n=6-8$ in each group. Statistical analyses were performed via two-tailed unpaired Student's t-test or two-way analysis of variance followed by Tukey's multiple comparison test. A p value $<0.05$ was considered statistically significant.

\section{RESULTS}

IL-6 expression in plasma and skeletal muscle induced by a single bout of exercise training

To first establish that the pattern of exercise training activated IL-6 production, we collected plasma and muscle tissue from C57BL/6 mice following a single bout of training and found that both the plasma IL-6 concentration and the muscle IL-6 mRNA level gradually increased from 1 hour post exercise training, reached a peak at approximately 8 hours following exercise and then gradually returned to basal levels in 48 hours (online supplemental figure S1A,B).
Chronic exercise training reduced HFD-induced body weight gain partially through IL-6

To evaluate the effects of IL-6 on HFD-induced metabolic dysfunction during exercise training, we first established a HFD-induced metabolic dysfunction model using IL-6 KO mice and WT mice and collected all subsequent data in these mice that were either sedentary or received exercise training five times per week for 8 weeks (figure 1A). We found that both HFD treatment and HFD+exercise treatment led to significantly elevated plasma IL-6 concentrations in WT mice $(\mathrm{p}<0.05)$, while no IL-6 was detected in any group of IL-6 $\mathrm{KO}$ mice (figure 1B). Consistently with previous studies, exercise significantly reduced long-term HFD-induced body weight gain in WT mice $(33.0 \%, \mathrm{p}<0.001)$. However, compared with WT mice, exercise could only partially decrease the HFDinduced body weight gain in IL-6 KO mice (16.4\%, $\mathrm{p}<0.05$ ) (figure $1 \mathrm{C}$ ). In our present study, food intake was not significantly affected by exercise training in either WT or IL-6 KO mice (online supplemental figure S2).

\section{IL-6 mediated exercise-induced VAT remodeling}

HFD-induced body weight gain is mainly due to the expansion of adipose tissue, especially VAT. ${ }^{16}{ }^{17}$ To investigate how IL-6 acts on VAT to regulate visceral fat lipid homeostasis during exercise, we compared histological and molecular changes between HFD fed WT mice and IL-6 KO mice in response to exercise training. HE staining showed that 8 weeks of treadmill running led to a marked reversal of HFD-induced visceral fat expansion in WT mice accompanied by smaller adipocyte size, whereas it had no obvious effects in IL-6 KO mice (figure 2A). Exercise significantly reduced HFD-upregulated visceral fat mass and adipocyte size in WT mice $(p<0.01)$, but had no effect on visceral fat mass loss or fat cell size in IL-6 KO mice (figure 2B,C). Considering that the alleviation of obesity is closely related to the lipolytic activities of adipose tissue, we detected the expression of two key genes involved in lipolysis, ATGL and HSL, and found that exercise could significantly upregulate the mRNA levels of ATGL $(p<0.01)$ and HSL $(p<0.05)$ in WT mice, but not in IL-6 KO mice (figure 2D,E). We further determined the protein expression of ATGL, pHSL and tHSL in VAT and found that exercise could also obviously increase the protein expression of ATGL $(\mathrm{p}<0.001)$ and markedly elevate the ratio of pHSL to tHSL $(p<0.001)$, while these changes were not observed in IL-6 KO mice. Meanwhile, the expression of ATGL and the ratio of $\mathrm{pHSL} / \mathrm{tHSL}$ in the exercise group of WT mice were found to be significantly higher than those in the exercise group of IL-6 KO mice $(\mathrm{p}<0.001)$ (figure $2 \mathrm{~F}, \mathrm{H})$.

\section{Exercise-induced thermogenesis of VAT depends on IL-6}

To determine whether IL-6 mediated the exercise-induced thermogenic effects of VAT, we detected the expression of genes involved in thermogenesis (PGC-1 $\alpha$, CYC1, TBX1, 
A

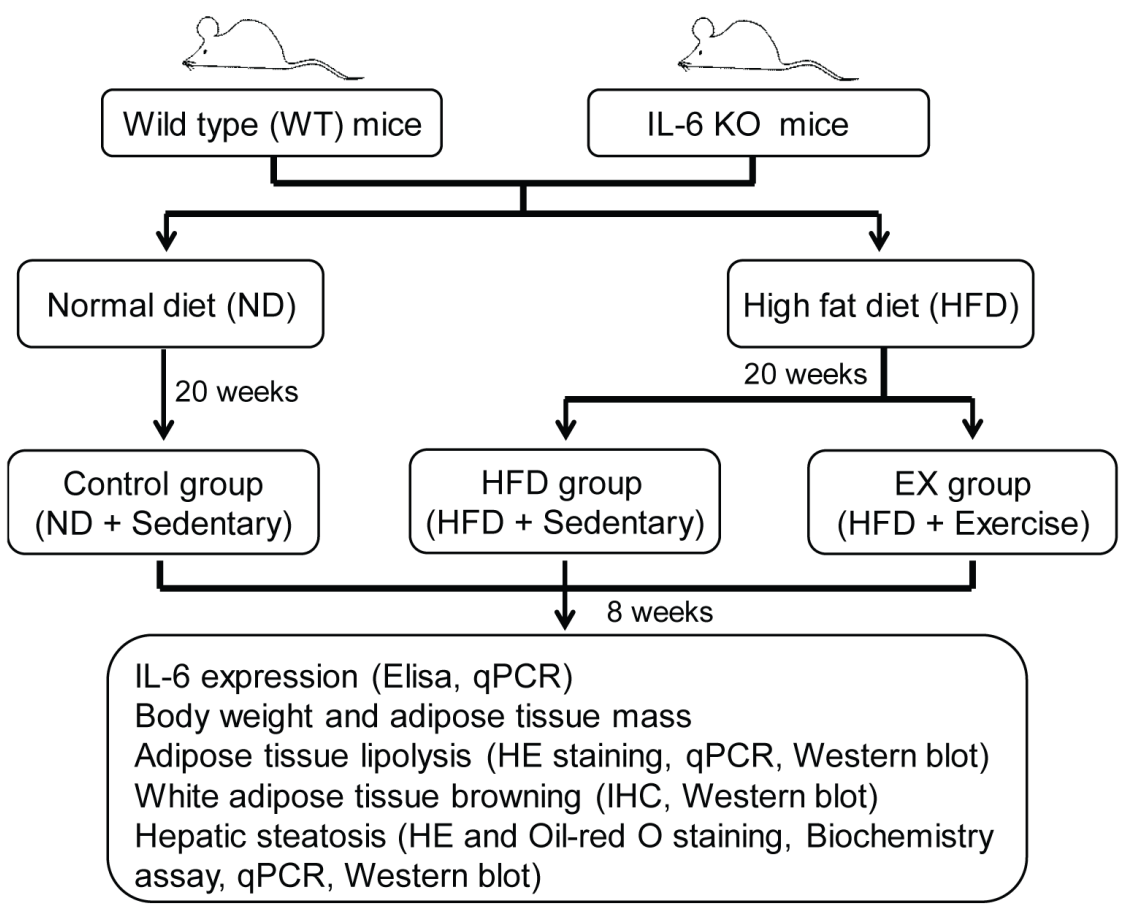

B

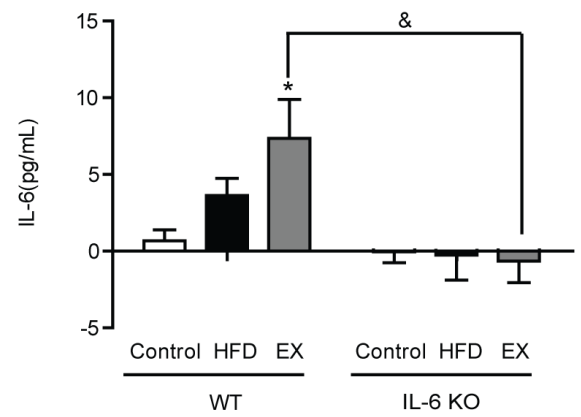

C

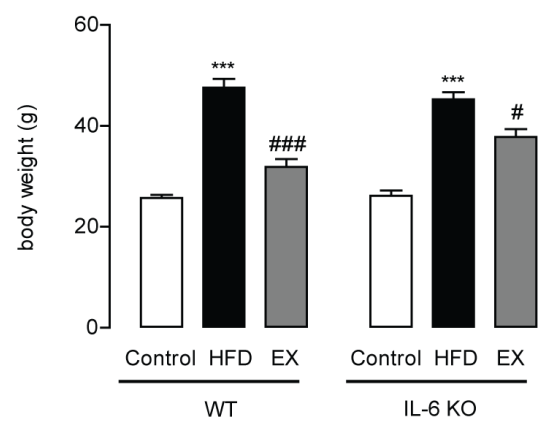

Figure 1 Effect of chronic exercise training on HFD-induced obesity in WT mice and IL-6 KO mice. (A) Study protocol of the effect of IL-6 on exercise-induced alleviation of adiposity and hepatic steatosis. (B) Plasma IL-6 concentrations in WT mice and IL-6 KO mice after 8 weeks of exercise training. (C) Body weight of WT mice and IL-6 KO mice treated for 8 weeks with exercise training. Data represent the mean $\pm \mathrm{SEM}, \mathrm{n}=6-8$ in each group. ${ }^{*} \mathrm{p}<0.05$ compared with the control group, ${ }^{* *} \mathrm{p}<0.001$ compared with the control group, \#p<0.05 compared with the HFD group, \#\#\#p<0.001 compared with the HFD group, \&p<0.05. EX, exercise; IHC, immunohistochemistry; IL-6, interleukin-6; KO, knockout.

PRDM16, CIDEA and CD137) and found that exercise significantly induced the mRNA levels of PGC- $1 \alpha$, CYC1, TBX1, PRDM16 and CIDEA in VAT of WT mice but not in IL-6 KO mice $(\mathrm{p}<0.05)$ (figure 3A). The above measured genes related to thermogenesis have been identified as markers of WAT browning, which are frequently linked to higher heat production and protection from diet-induced obesity. Previous studies demonstrated that PGC-1 $\alpha$ is a key protein during WAT browning. Using immunostaining and western blot methods, we further confirmed that the protein levels of PGC- $1 \alpha$ were significantly upregulated after exercise training in WT mice $(\mathrm{p}<0.05)$, while no obvious changes were observed in IL-6 KO mice (figure 3B and $\mathrm{C}$ ).

\section{Exercise training promotes subcutaneous fat lipolysis and thermogenesis via IL-6}

Consistently with what we discovered in VAT, treadmill training also markedly alleviated subcutaneous fat expansion in WT mice, but had no effects in IL-6 KO mice as identified by HE staining, fat mass weight and adipocyte size quantitation (figure $4 \mathrm{~A}-\mathrm{C}$ ). These effects may be due to a significant induction of ATGL, HSL, PGC- $1 \alpha$ and PRDM16 mRNA expression after exercise training in WT mice $(p<0.05)$, which was not observed in IL-6 KO mice (figure 4D). Protein expression and distribution analysis revealed that PGC- $1 \alpha$ protein levels in subcutaneous adipose tissue (SAT) were considerably elevated after treadmill training in WT mice $(\mathrm{p}<0.05)$. In contrast, no differences in the protein expression of PGC- $1 \alpha$ levels 
A
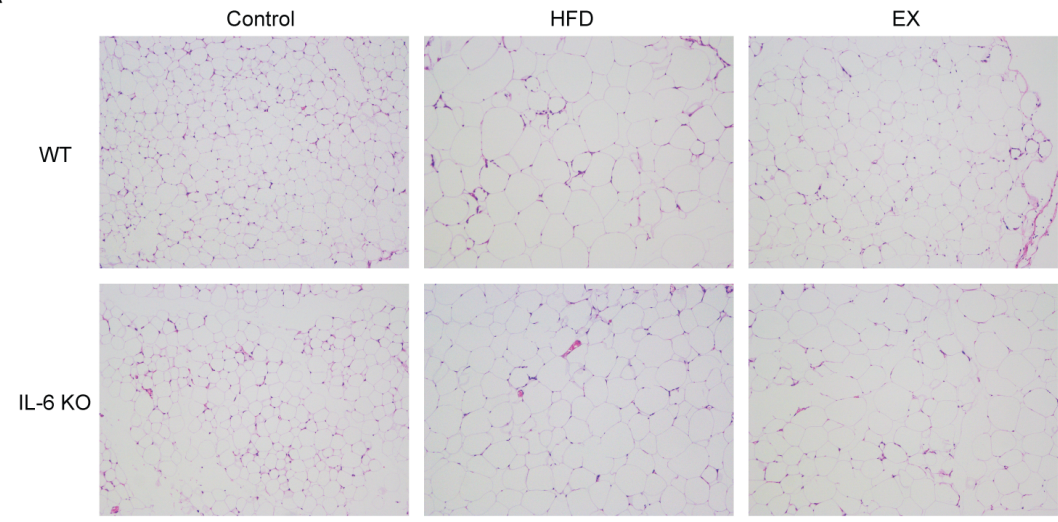

D
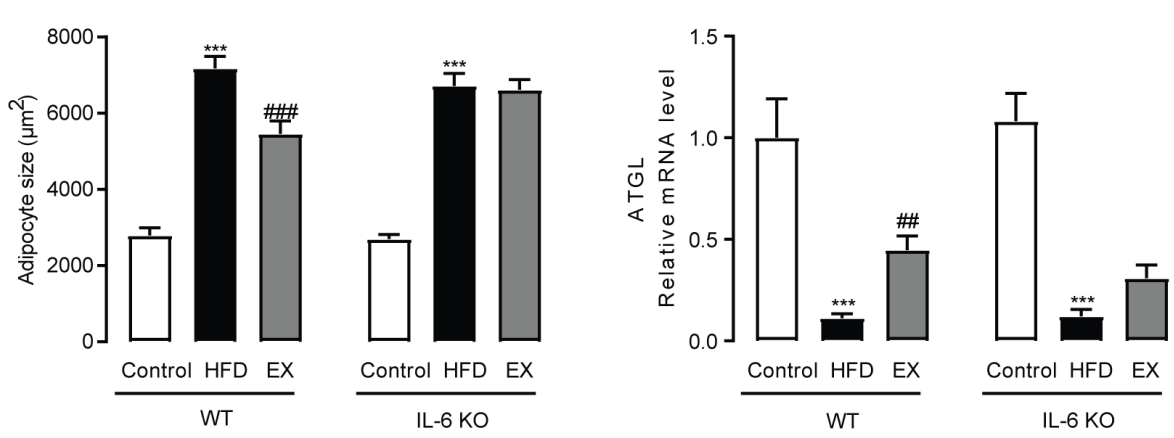

F

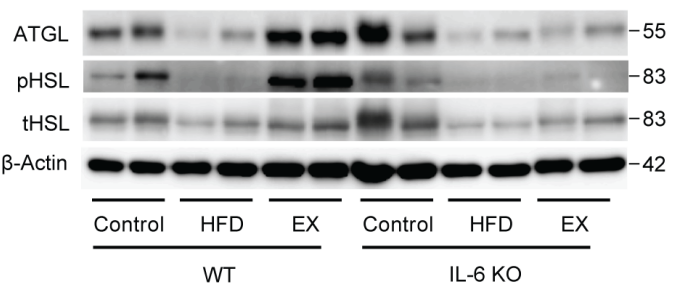

G

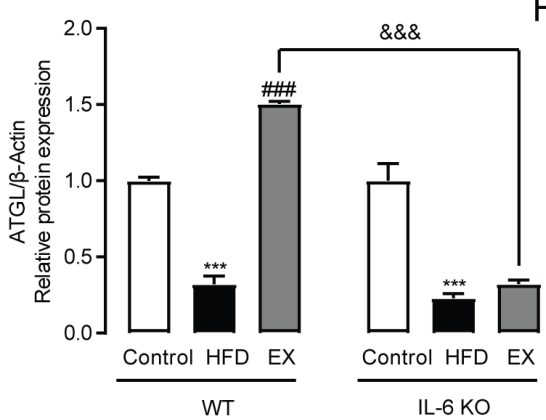

B

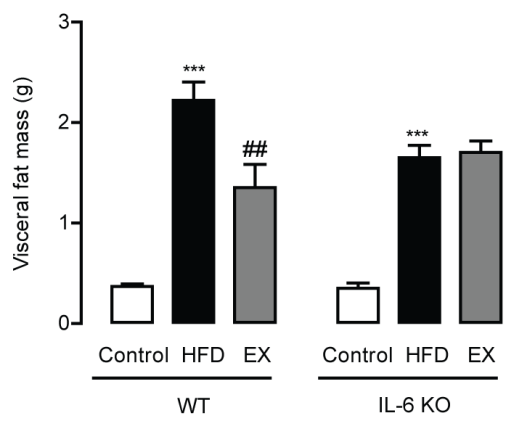

E

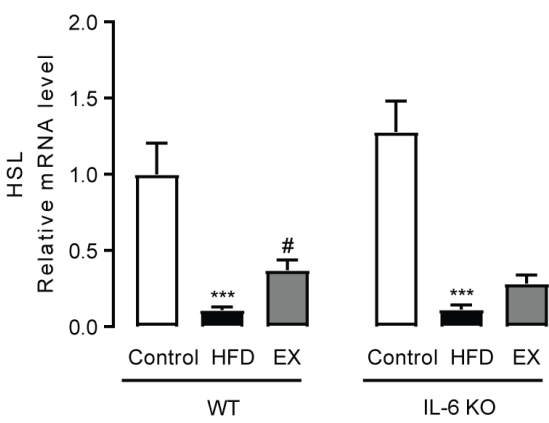

$\mathrm{H}$

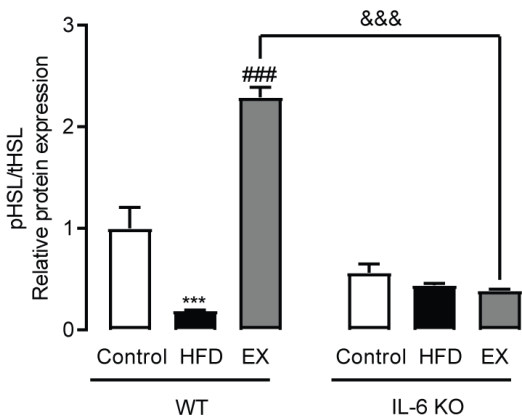

Figure 2 Exercise training regulates visceral fat expansion in WT mice and IL-6 KO mice. (A) HE staining (original magnification, $\times 400$ ) of visceral adipose tissues from WT mice and IL-6 KO mice following 8 weeks of exercise training. visceral fat mass (B) and average adipocyte size (C) in WT mice and IL-6 KO mice treated for 8 weeks with exercise training. ATGL (D) and HSL (E) mRNA expression in visceral adipose tissues from WT mice and IL-6 KO mice. (F-H) Protein expression levels of ATGL, pHSL and tHSL in visceral adipose tissues from WT mice and IL-6 KO mice following 8 weeks of exercise training. Data represent the mean $\pm S E M, n=6-8$ in each group. ${ }^{* *} p<0.001$ compared with the control group, $\# p<0.05$ compared with the HFD group, \#\#p<0.01 compared with the HFD group, \#\#\#p<0.001 compared with the HFD group, \&\&\&p<0.001. EX, exercise; HFD, high-fat diet; IL-6, interleukin-6; KO, knockout; WT, wild type.

were observed between HFD-fed mice that remained sedentary and those that received exercise training in IL-6 KO mice (figure 4E and F).

\section{Exercise training alleviated HFD-induced hepatic steatosis partially through IL-6}

To evaluate the effect of IL-6 on HFD-induced hepatic steatosis during exercise training, we recorded the relative liver weights, determined hepatic TG levels and plasma ALT levels through biochemical assays, evaluated liver histological changes by HE staining and measured hepatic lipid droplet formation with Oil red O staining. Consistent with elevated body weight and adipose tissue mass, HFD induced serious hepatic steatosis in both WT mice and IL-6 KO mice as supported by increased liver weight, large areas of lipid accumulation and obviously elevated hepatic TG $(\mathrm{p}<0.001, \mathrm{p}<0.001)$ and plasma ALT $(p<0.05, p<0.01)$ levels. After 8 weeks of treadmill running, we found that exercise training significantly reduced hepatocyte ballooning (figure 5A), Oil red O-stained area (figure $5 \mathrm{~B}$ ), liver weight (figure $5 \mathrm{C}$ ), hepatic TG $(\mathrm{p}<0.001)$ and plasma ALT $(\mathrm{p}<0.01)$ levels in WT mice fed a HFD (figure 5D and E). The benefits of exercise in WT mice were markedly powerful in reversing the degree of hepatic steatosis of the HFD group to the same 
A
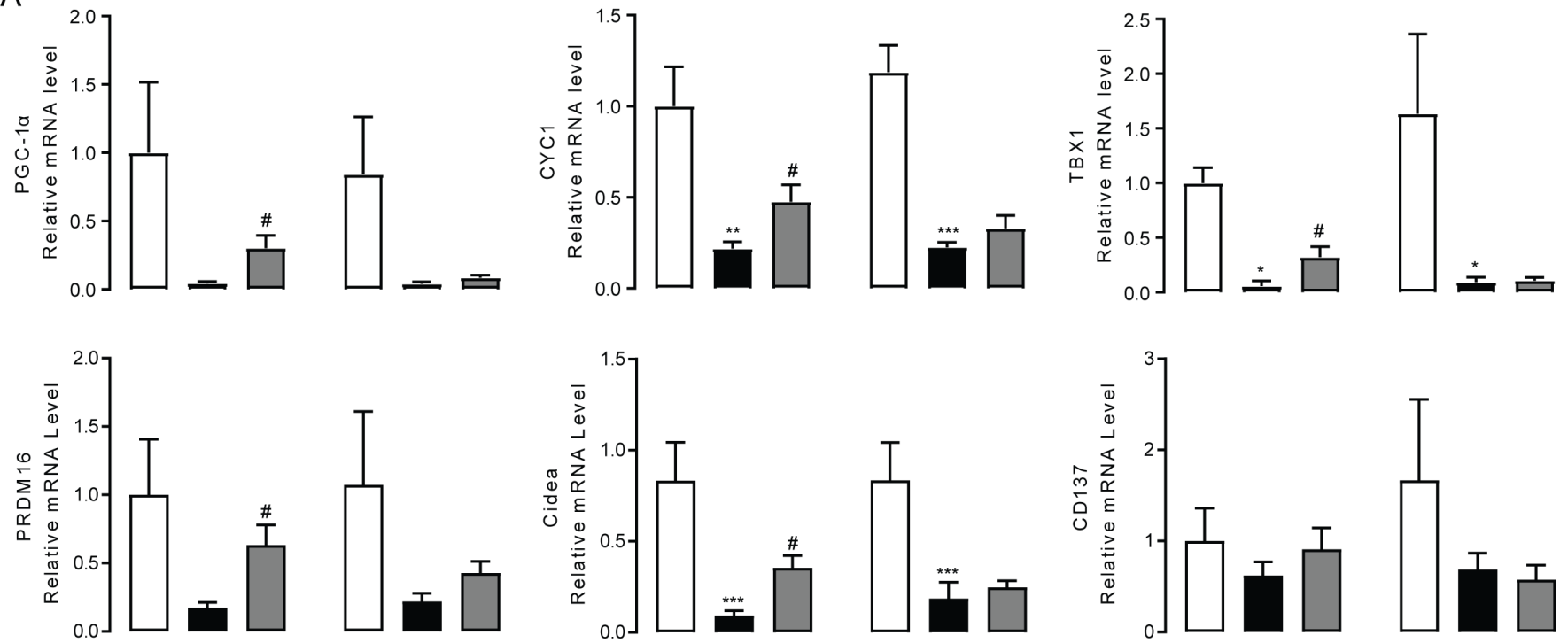

B

C
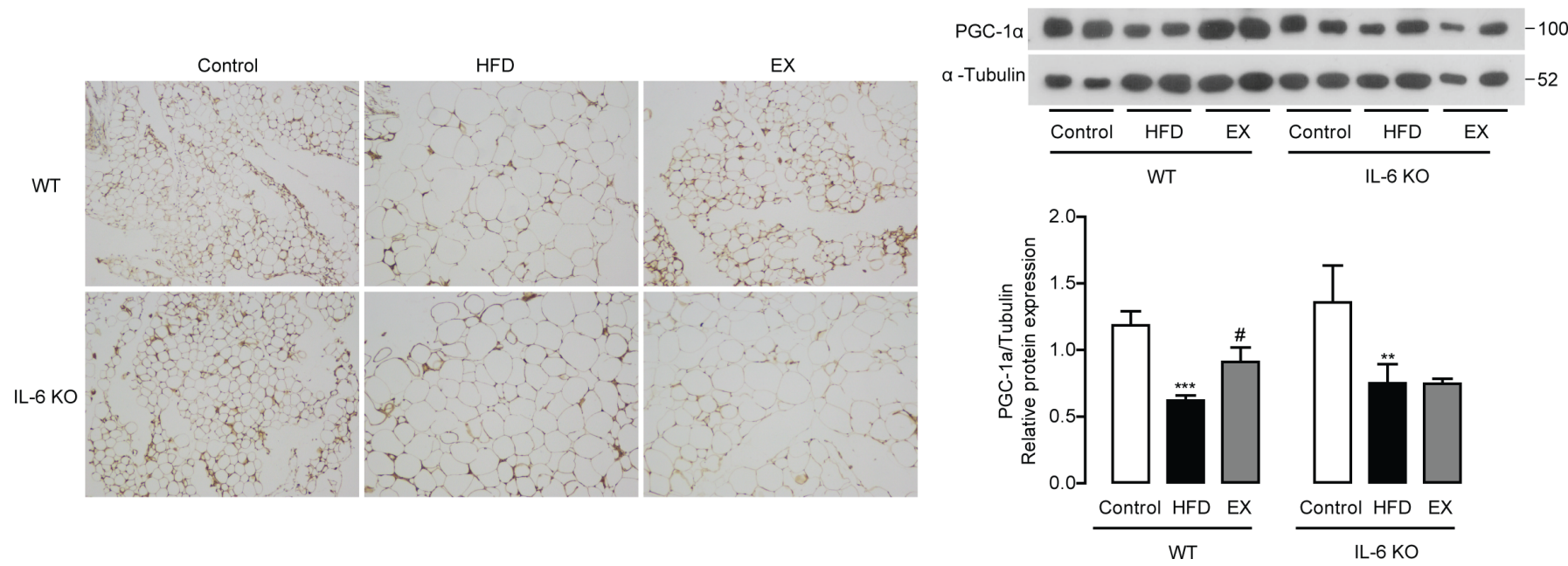

Figure 3 Effect of exercise training on thermogenesis in visceral adipose tissue from WT mice and IL-6 KO mice. (A) PGC$1 \alpha$, CYC1, TBX1, PRDM16, CIDEA and CD137 mRNA expression levels in visceral adipose tissues from WT mice and IL-6 KO mice. (B) Immunochemistry staining (original magnification, $\times 100$ ) of PGC-1 $\alpha$ in visceral adipose tissues from WT mice and IL-6 $\mathrm{KO}$ mice following 8 weeks of exercise training. (C) Protein expression levels of PGC- $1 \alpha$ in visceral adipose tissues from WT mice and IL-6 KO mice following 8 weeks of exercise training. Data represent the mean $\pm S E M, n=6-8$ in each group. ${ }^{*} p<0.05$ compared with the control group, ${ }^{* *} \mathrm{p}<0.01$ compared with the control group, ${ }^{* * *} \mathrm{p}<0.001$ compared with the control group, $\# p<0.05$ compared with the HFD group. EX, exercise; HFD, high-fat diet; IL-6, interleukin-6; KO, knockout; WT, wild type.

degree as the control group fed a normal diet. However, exercise training in IL-6 KO mice could not reproduce similar effects as in WT mice. Although compared with the HFD group, the HFD+exercise group showed markedly decreased liver weight, less lipid accumulation and reduced plasma TG $(\mathrm{p}<0.001)$ and ALT $(\mathrm{p}<0.05)$ levels in IL-6 KO mice, livers from the HFD+exercise group in IL-6 KO mice still exhibited much more lipid droplet accumulation than those of WT mice (figure $5 \mathrm{~A}$ and $\mathrm{B}$ ). In addition, liver weight (figure 5C), hepatic TG and plasma ALT levels in IL-6 KO mice subjected to treadmill running remained much higher than those of WT mice $(\mathrm{p}<0.05)$ (figure 5D and $\mathrm{E})$. These results suggested that IL-6 partially mediated the effect of exercise training on HFD-induced hepatic steatosis.
Furthermore, to determine the mechanisms by which IL-6 mediates the effect of treadmill running on hepatic steatosis, we measured the mRNA levels of genes associated with lipogenesis (SREBP-1c, ACC-1, FASN, SCD-1 and PPAR- $\gamma$ ) and fatty acid $\beta$-oxidation (PPAR- $\alpha$ and CPT-1) (online supplemental figure S3). We noticed no differences in these genes between WT mice and IL-6 KO mice receiving exercise training except for PPAR- $\gamma$, which displayed lower expression in WT mice than in IL-6 KO mice. We further noted that hepatic protein levels of PPAR- $\gamma$ in the HFD group were significantly increased in both WT mice and IL-6 KO mice compared with the control group. After exercise training, hepatic PPAR- $\gamma$ protein levels were downregulated significantly in WT mice but not in IL-6 KO mice (figure 5F and G). These 
A

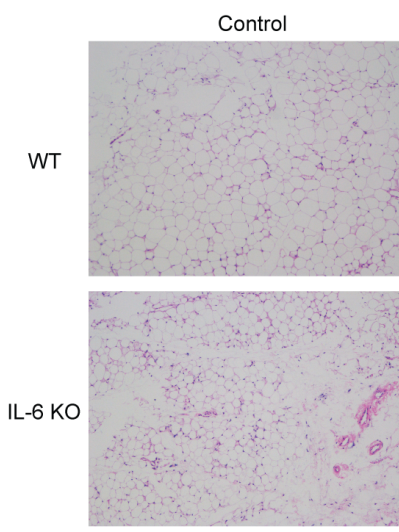

$\mathrm{D}$
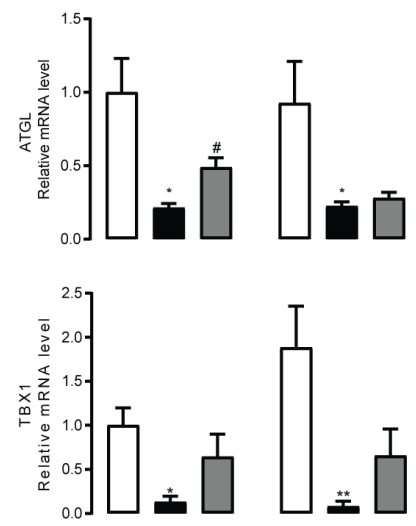

$\mathrm{E}$

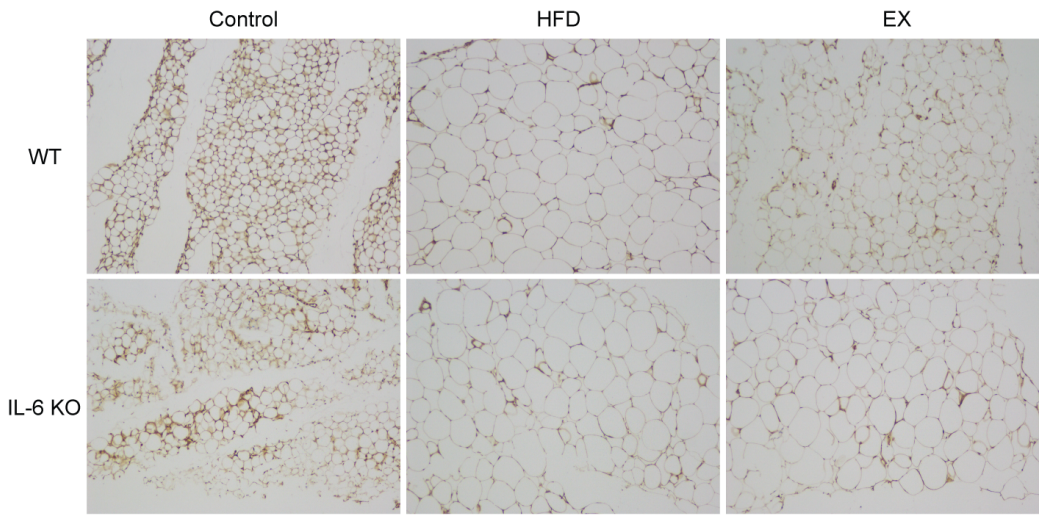

EX
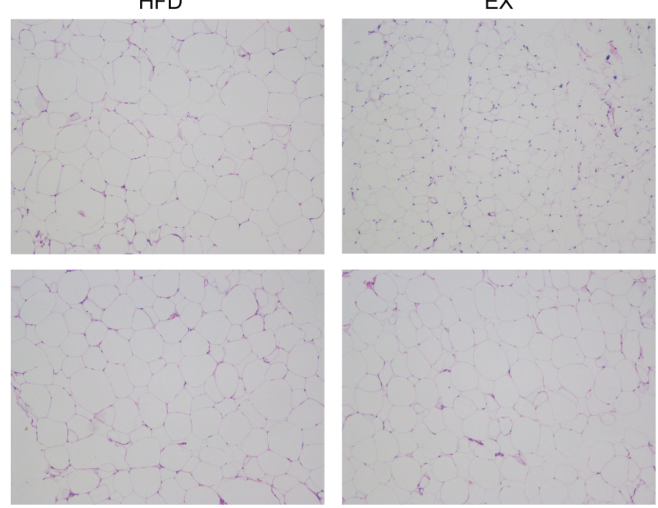

B

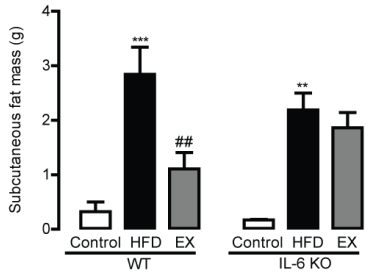

C

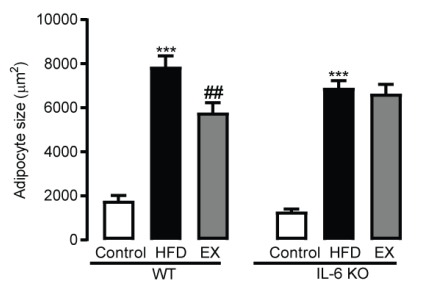

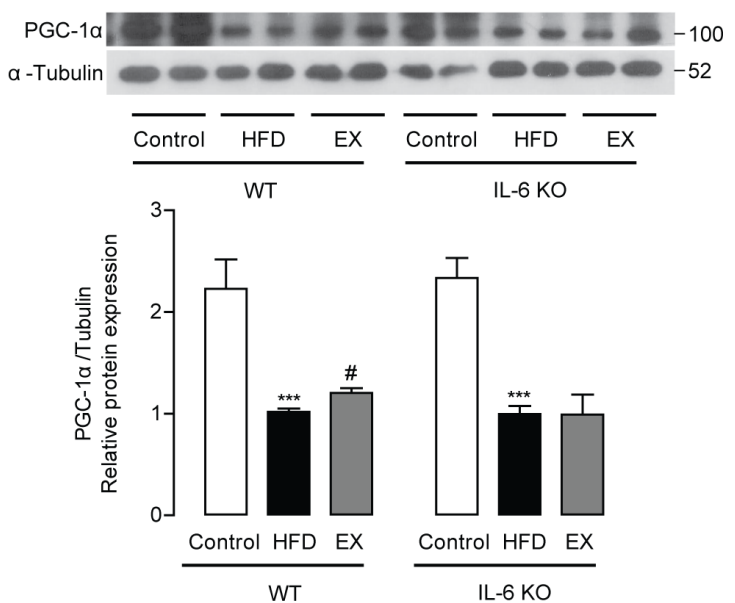

Figure 4 Exercise training regulates subcutaneous fat lipolysis and thermogenesis in WT mice and IL-6 KO mice. (A) HE staining (original magnifications, $\times 400$ ) of subcutaneous adipose tissues from WT mice and IL-6 KO mice following 8 weeks of exercise training. Subcutaneous fat mass (B) and average adipocyte size (C) in WT mice and IL-6 KO mice treated for 8 weeks with exercise training. (D) ATGL, HSL, PGC-1 $\alpha$, CYC1, TBX1, PRDM16, CIDEA and CD147 mRNA expression in visceral adipose tissues from WT mice and IL-6 KO mice. Immunochemistry staining (original magnifications, $\times 100)$ of PGC-1 $\alpha(E)$ in subcutaneous adipose tissues from WT mice and IL-6 KO mice following 8 weeks of exercise training. (F) Protein expression levels of PGC- $1 \alpha$ in subcutaneous adipose tissues from WT mice and IL-6 KO mice following 8 weeks of exercise training. Data represent the mean \pm SEM, $n=6-8$ in each group. ${ }^{*} p<0.05$ compared with the control group, ${ }^{* *} p<0.01$ compared with the control group, ${ }^{\star \star *} p<0.001$ compared with the control group, $\# p<0.05$ compared with the HFD group, \#\# $p<0.01$ compared with the HFD group. EX, exercise; HFD, high-fat diet; IL-6, interleukin-6; KO, knockout; WT, wild type

findings indicated that the IL-6-dependent antisteatosis property of exercise could be potentially mediated by the regulation of PPAR- $\gamma$.

\section{DISCUSSION}

In humans, physical activity is a commonly recommended approach for treating patients with metabolic 
A

HFD

EX

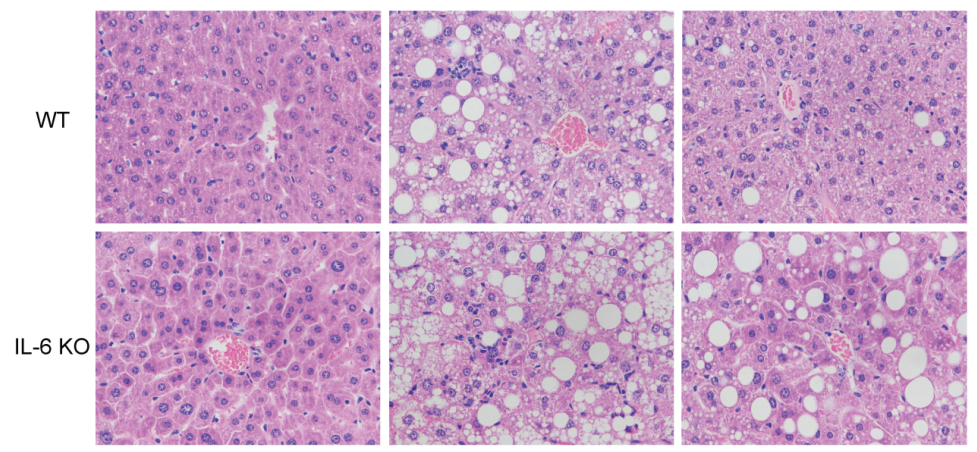

B

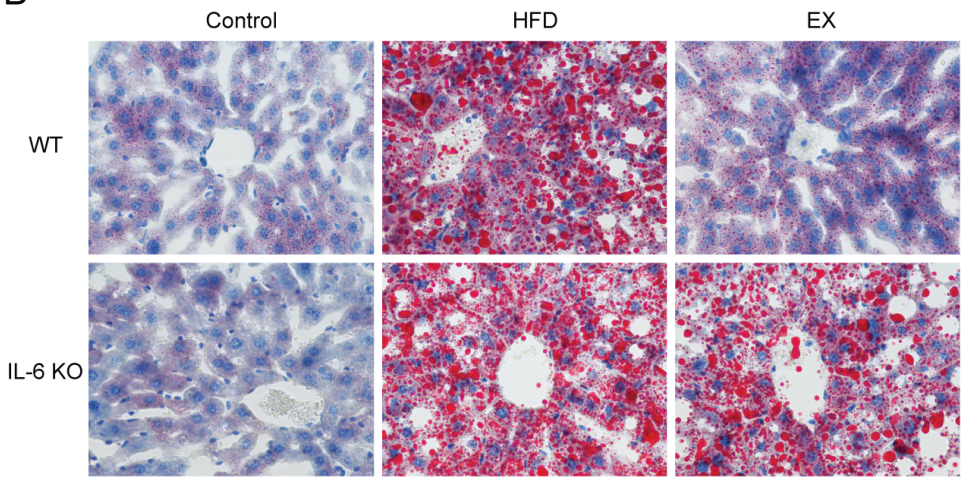

C

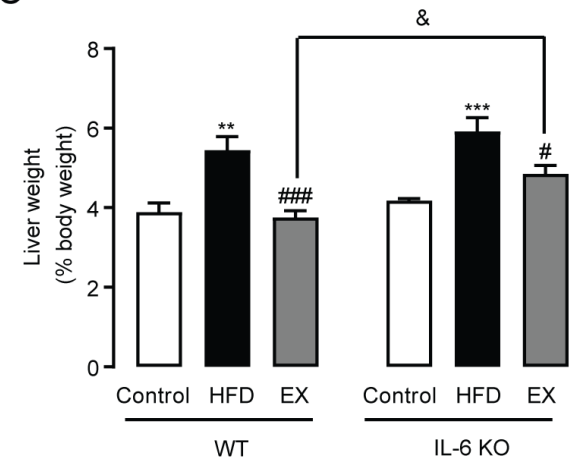

$\mathrm{F}$

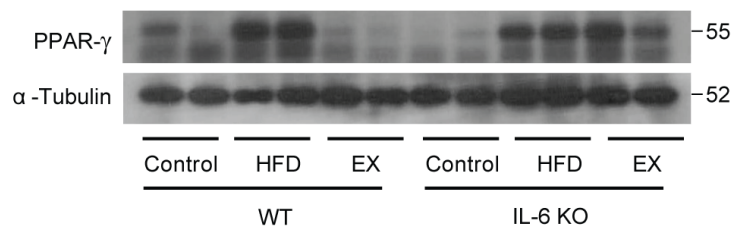

$\mathrm{E}$

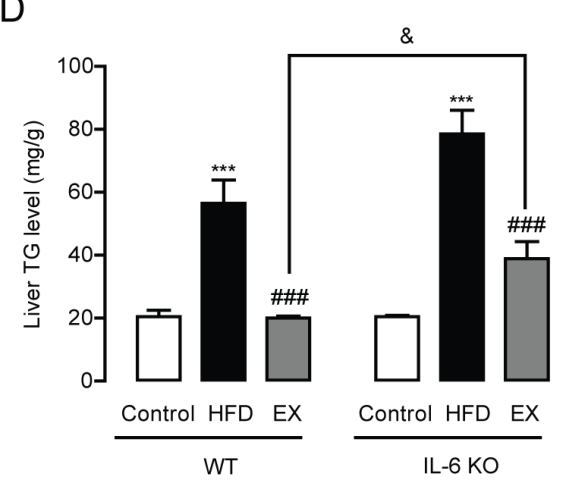

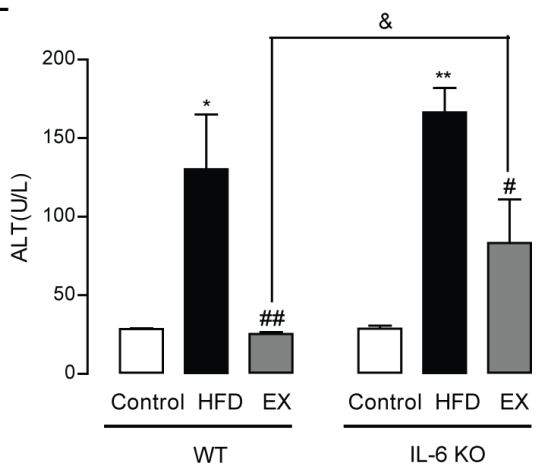

G

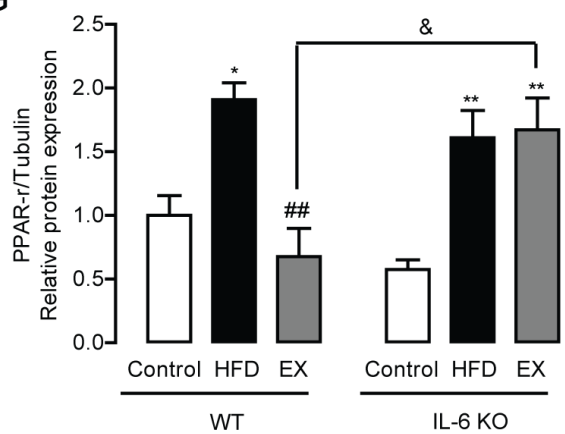

Figure 5 Effect of exercise training on HFD-induced hepatic steatosis in WT mice and IL-6 KO mice. (A) HE staining (original magnifications, $\times 400$ ) and $(B)$ Oil red O staining (original magnification, $\times 400$ ) of liver tissues from WT mice and IL-6 KO mice following 8 weeks of exercise training. (C) Liver weight, (D) liver TG and (E) plasma ALT levels in WT mice and IL-6 KO mice following 8 weeks of exercise training. $(F, G)$ Protein expression levels of hepatic PPAR- $\gamma$ in WT mice and IL-6 KO mice following 8 weeks of exercise training. Data represent the mean $\pm S E M, n=6-8$ in each group. ${ }^{*} p<0.05$ compared with the control group, ${ }^{* *} p<0.01$ compared with the control group, ${ }^{* * *} p<0.001$ compared with the control group, $\# p<0.05$ compared with the HFD group, \#\#p<0.01 compared with the HFD group, \#\#\#p<0.001 compared with the HFD group, \&p<0.05. ALT, aspartate transaminase; EX, exercise; HFD, high-fat diet; IL-6, interleukin-6; KO, knockout; PPAR- $\gamma$, proliferator-activated receptor gamma; TG, triglyceride; WT, wild type. 
dysfunctions. Here, we show that treadmill training induced an exponential elevation of plasma IL-6 levels in WT mice. Taking previous studies into account, we speculate that the large amount of IL-6 detected in the circulation during exercise may mainly be derived from skeletal muscle. ${ }^{18}$ Mounting research has explained how IL-6 is released from skeletal muscles during exercise training and that it is now considered to be secreted as a result of muscle glycogen depletion. ${ }^{19}$ The beneficial effects of exercise training on adiposity and hepatic steatosis have been widely demonstrated by various previous reports. ${ }^{2021}$ Our data were consistent with previous studies showing the beneficial effects of treadmill running on decreased visceral fat and subcutaneous fat mass as well as reduced hepatic steatosis in HFD-induced obese WT mice. However, very limited studies in the past have investigated the molecular mechanisms underlying the benefits of exercise on system health.

The relationship between IL- 6 and the accumulation of adipose tissue is controversial. Several studies have demonstrated that global deletion of IL- 6 in mice results in the accumulation of adipose tissue, ${ }^{5}$ while other studies have revealed that IL-6 does not affect the development of obesity. ${ }^{22}$ Our study detected no significant difference between IL-6 KO mice and WT mice fed a HFD under sedentary conditions. However, we noted that exercise had a more significant downregulation effect on VAT mass and SAT mass in WT mice than in IL-6 KO mice. This may be because IL-6 production in resting mice is too low to exert real physiological effects. Excessive accumulation of visceral fat is associated with insulin resistance and an increased risk of type 2 diabetes, while the enlargement of SAT is associated with insulin sensitivity and a decreased risk of type 2 diabetes. ${ }^{23}$ Our present study found that IL- 6 contributes to both visceral and subcutaneous fat loss during exercise training, which may explain the phenomenon in which IL-6 has no effect on exercise-improved insulin resistance (online supplemental figure $\mathrm{S} 4$ ).

Recent studies have reported that prolonged cold exposure or $\beta 3$-adrenergic receptor agonist treatment could stimulate adipocytes in white adipose tissue (WAT) to transdifferentiate into cells similar to adipocytes in brown adipose tissue, which was defined as the 'browning' process. The transcriptional coactivator PGC- $1 \alpha$ and the mitochondrial uncoupling protein UCP-1 are widely recognized as adipocyte browning markers. Both human and rodent studies have indicated that exercise training could increase the expression of PGC- $1 \alpha$ and UCP-1. ${ }^{24} 25$ Irisin, another well-known myokine, has been reported to drive brown fat-like development of WAT during exercise by stimulating PGC-1 $\alpha$ and UCP-1 expression. ${ }^{26}$ Previous work has shown that IL-6 could induce the expression of PGC-1 $\alpha$ in 3T3-L1 adipocytes and cultured VAT, but not in cultured SAT. ${ }^{27} 28$ A previous study demonstrated that 5 weeks of exercise training increased UCP-1 expression in SAT through IL-6 but had no effect on PGC- $1 \alpha$ expression. ${ }^{29}$ In the present study, we found that exercise training significantly increased the protein expression of PGC- $1 \alpha$ in both VAT and SAT from WT mice, which was consistent with previous studies employing the same exercise training program. ${ }^{30}$ Interestingly, these pro-browning effects were not observed in IL-6 KO mice. We also tried to determine the protein expression of UCP-1 through western blotting in the present study. Unfortunately, we failed to observe the specific band of UCP-1 in either VAT or SAT using brown adipose tissue as the positive control (data not shown). Thus, the findings of our present study identified IL-6 as a mediator of exercise-induced adipose tissue browning. However, further studies are still needed to determine whether UCP-1 plays a key role in the IL-6mediated thermogenic effects during exercise.

Several previous studies have reported the effects of IL-6 on liver metabolism during exercise. For example, longterm exercise training influenced hepatic cytochrome P450 expression through skeletal muscle-released IL-6. ${ }^{31}$ Skeletal muscle IL-6 could regulate hepatic glucose metabolism during prolonged exercise. ${ }^{32}$ Our recent clinical study demonstrated that both moderate-intensity and highintensity exercise significantly reduced the intrahepatic triglyceride content. ${ }^{33}$ Of note, our present study demonstrates that the protective effects of exercise against obesityinduced hepatic steatosis are mediated partially by IL-6. PPAR- $\gamma$ signaling plays an important role in lipogenic and fatty acid transport. Liver-specific PPAR- $\gamma$ knockdown mice are resistant to HFD-induced liver steatosis primarily through suppressing fatty acid uptake and esterification. ${ }^{34}$ The deficiency of hepatic PPAR- $\gamma$ also dramatically decreased liver TG levels in leptin-deficient $(\mathrm{ob} / \mathrm{ob})$ obese mice. ${ }^{35} \mathrm{~A}$ previous manuscript revealed a role of IL- 6 in stimulating PPAR- $\gamma$ expression elevation after a single bout of treadmill running in SAT. ${ }^{36}$ However, no study has focused on the role of IL-6 in PPAR- $\gamma$ expression in the liver during or after treadmill training. Our current study showed that PPAR- $\gamma$ is the only lipogenic gene dramatically suppressed by IL-6, suggesting that PPAR- $\gamma$ signaling regulated by IL- 6 may account for most of the effects of exercise on intrahepatic TG content reduction.

The major limitation of our present study is that none of the animal experiments were performed at thermal neutrality, which has been proven to be an effective method to reduce the discrepancy in metabolism-related studies between humans and mice. Housing temperature has been reported to affect acute and chronic exercise-induced markers of mitochondrial biogenesis and browning of WAT, which were reduced in mice housed at thermoneutrality. ${ }^{37}$ Consistently with this, another recent study also highlighted the importance of housing temperature on influencing exerciseinduced metabolic improvements, which was partially dependent on blunted molecular adaptations to exercise training in adipose tissue.$^{38}$ Of note, compared with visceral WAT, both studies emphasized a more pronounced difference in subcutaneous WAT adaption to exercise training between mice housed at room temperature and thermoneutrality. The beneficial effects of exercise training on visceral WAT seemed to be unaffected by thermoneutrality. ${ }^{37} 38$ 
In our present study, IL-6 was found to be involved in the regulation of both visceral and subcutaneous WAT during exercise training. Therefore, it would be logical to predict that the function of IL-6 in ameliorating visceral WAT accumulation may persist in mice receiving exercise training at thermoneutrality. Taken together, it would be of interest to further study the effects of IL-6 in exercised animals housed at thermoneutrality compared with room temperature.

In summary, we exposed WT and IL-6 KO mice to HFD and exercise training in the present study and observed a role of IL-6 in the exercise-induced metabolic benefits on HFDinduced adiposity and hepatic steatosis. Moreover, we identified IL-6 as the key molecule accounting for the pro-lipolytic and thermogenic effects of exercise in both visceral and SAT. The beneficial effects of exercise on obesity-related hepatic steatosis may be dependent on IL-6-suppressed PPAR- $\gamma$ signaling. It is possible that IL-6 could mimic the beneficial effects of exercise on obesity and non-alcoholic fatty liver disease, which would function as an effective strategy for the treatment of these metabolic dysfunctions.

Acknowledgements We thank Professor Xiaoying Li (Fudan University, Shanghai, China) for assistance in study design.

Contributors LL, YZ and XL designed the experiments. LL, CH, HY, XZ and DW performed the experiments. $\mathrm{CH}, \mathrm{CM}, \mathrm{JL}$ and $\mathrm{XZ}$ analyzed the data. $\mathrm{YZ}$ and $\mathrm{XL}$ contributed reagents and materials. $\mathrm{LL}, \mathrm{CH}, \mathrm{YZ}$ and $\mathrm{XL}$ wrote the manuscript. All authors approved the final version of the manuscript.

Funding This study was supported by the National Natural Science Foundation of China (No 81570770, No 81870606); the Natural Science Foundation of Fujian Province (No 15J01552); the Natural Science Foundation of Zhejiang Province (No LQ21H030002) and the Ningbo Natural Science Foundation (No 2019A610209).

Competing interests None declared.

Patient consent for publication Not required.

Ethics approval All animal experiments performed were approved by the Committee for Animal Research at Xiamen University.

Provenance and peer review Not commissioned; externally peer reviewed.

Data availability statement All data relevant to the study are included in the article or uploaded as supplemental information.

Supplemental material This content has been supplied by the author(s). It has not been vetted by BMJ Publishing Group Limited (BMJ) and may not have been peer-reviewed. Any opinions or recommendations discussed are solely those of the author(s) and are not endorsed by BMJ. BMJ disclaims all liability and responsibility arising from any reliance placed on the content. Where the content includes any translated material, BMJ does not warrant the accuracy and reliability of the translations (including but not limited to local regulations, clinical guidelines, terminology, drug names and drug dosages), and is not responsible for any error and/or omissions arising from translation and adaptation or otherwise.

Open access This is an open access article distributed in accordance with the Creative Commons Attribution Non Commercial (CC BY-NC 4.0) license, which permits others to distribute, remix, adapt, build upon this work non-commercially, and license their derivative works on different terms, provided the original work is properly cited, appropriate credit is given, any changes made indicated, and the use is non-commercial. See: http://creativecommons.org/licenses/by-nc/4.0/.

\section{ORCID iDs}

Long Li http://orcid.org/0000-0001-9103-3905

Xuejun Li http://orcid.org/0000-0001-6940-3180

\section{REFERENCES}

1 Geng L, Liao B, Jin L, et al. Exercise alleviates obesity-induced metabolic dysfunction via enhancing FGF21 sensitivity in adipose tissues. Cell Rep 2019;26:2738-52.
2 Pedersen BK. Physical activity and muscle-brain crosstalk. Nat Rev Endocrinol 2019;15:383-92.

3 Pereira RM, Rodrigues KCdaC, Anaruma CP, et al. Short-Term strength training reduces gluconeogenesis and NAFLD in obese mice. J Endocrinol 2019;241:59-70.

4 Kujala UM. Evidence on the effects of exercise therapy in the treatment of chronic disease. Br J Sports Med 2009;43:550-5.

5 Wallenius $\mathrm{V}$, Wallenius $\mathrm{K}$, Ahrén $\mathrm{B}$, et al. Interleukin-6-deficient mice develop mature-onset obesity. Nat Med 2002;8:75-9.

6 Ellingsgaard H, Hauselmann I, Schuler B, et al. Interleukin-6 enhances insulin secretion by increasing glucagon-like peptide- 1 secretion from L cells and alpha cells. Nat Med 2011;17:1481-9.

7 van Hall G, Steensberg A, Sacchetti M, et al. Interleukin-6 stimulates lipolysis and fat oxidation in humans. $J$ Clin Endocrinol Metab 2003;88:3005-10.

8 Nishimoto N, Kanakura Y, Aozasa K, et al. Humanized antiinterleukin-6 receptor antibody treatment of multicentric Castleman disease. Blood 2005;106:2627-32.

9 Ma Y, Gao M, Sun H, et al. Interleukin-6 gene transfer reverses body weight gain and fatty liver in obese mice. Biochim Biophys Acta 2015;1852:1001-11.

10 Li L, Duan C, Zhao Y, et al. Preventive effects of interleukin-6 in lipopolysaccharide/D-galactosamine induced acute liver injury via regulating inflammatory response in hepatic macrophages. Int Immunopharmacol 2017:51:99-106.

11 Görgens SW, Eckardt K, Jensen J, et al. Exercise and regulation of adipokine and myokine production. Prog Mol Biol Trans/ Sci 2015;135:313-36.

12 Pedersen BK, Fischer CP. Beneficial health effects of exercise--the role of IL-6 as a myokine. Trends Pharmacol Sci 2007;28:152-6.

13 Pedersen BK, Febbraio MA. Muscles, exercise and obesity: skeletal muscle as a secretory organ. Nat Rev Endocrinol 2012;8:457-65.

14 Wedell-Neergaard A-S, Lang Lehrskov L, Christensen RH, et al. Exercise-Induced changes in visceral adipose tissue mass are regulated by IL-6 signaling: a randomized controlled trial. Cell Metab 2019;29:844-55.

15 Li L, Yin H, Zhao Y, et al. Protective role of puerarin on LPS/D-Gal induced acute liver injury via restoring autophagy. Am J Trans/ Res 2018:10:957-65.

16 Gawronska-Kozak B, Staszkiewicz J, Gimble JM, et al. Recruitment of fat cell precursors during high fat diet in C57BL/6J mice is fat depot specific. Obesity 2014;22:1091-102.

17 Julien SG, Kim S-Y, Brunmeir R, et al. Narciclasine attenuates diet-induced obesity by promoting oxidative metabolism in skeletal muscle. PLoS Biol 2017;15:e1002597.

18 Morettini M, Palumbo MC, Sacchetti M, et al. A system model of the effects of exercise on plasma interleukin- 6 dynamics in healthy individuals: role of skeletal muscle and adipose tissue. PLoS One 2017; 12:e0181224.

19 Hojman P, Brolin C, Nørgaard-Christensen N, et al. II-6 release from muscles during exercise is stimulated by lactate-dependent protease activity. Am J Physiol Endocrinol Metab 2019;316:E940-7.

20 Thompson D, Karpe F, Lafontan M, et al. Physical activity and exercise in the regulation of human adipose tissue physiology. Physiol Rev 2012;92:157-91.

21 Sargeant JA, Gray LJ, Bodicoat DH, et al. The effect of exercise training on intrahepatic triglyceride and hepatic insulin sensitivity: a systematic review and meta-analysis. Obes Rev 2018;19:1446-59.

22 Di Gregorio GB, Hensley L, Lu T, et al. Lipid and carbohydrate metabolism in mice with a targeted mutation in the IL- 6 gene: absence of development of age-related obesity. Am J Physiol Endocrinol Metab 2004;287:E182-7.

23 Tran TT, Yamamoto Y, Gesta S, et al. Beneficial effects of subcutaneous fat transplantation on metabolism. Cell Metab 2008; 7:410-20.

24 Stanford KI, Middelbeek RJW, Goodyear LJ. Exercise effects on white adipose tissue: Beiging and metabolic adaptations. Diabetes 2015;64:2361-8.

25 Stanford KI, Middelbeek RJW, Townsend KL, et al. A novel role for subcutaneous adipose tissue in exercise-induced improvements in glucose homeostasis. Diabetes 2015;64:2002-14.

26 Boström P, Wu J, Jedrychowski MP, et al. A PGC1- $\alpha$-dependent myokine that drives brown-fat-like development of white fat and thermogenesis. Nature 2012;481:463-8.

27 Ji C, Chen X, Gao C, et al. II-6 induces lipolysis and mitochondrial dysfunction, but does not affect insulin-mediated glucose transport in 3T3-L1 adipocytes. J Bioenerg Biomembr 2011;43:367-75.

28 Wan Z, Perry CGR, Macdonald T, et al. II-6 is not necessary for the regulation of adipose tissue mitochondrial content. PLoS One 2012; : e51233 
29 Knudsen JG, Murholm M, Carey AL, et al. Role of IL-6 in exercise training- and cold-induced UCP1 expression in subcutaneous white adipose tissue. PLoS One 2014;9:e84910.

30 Aldiss P, Betts J, Sale C, et al. Exercise-induced 'browning' of adipose tissues. Metabolism 2018;81:63-70.

31 Knudsen JG, Bertholdt L, Gudiksen A, et al. Skeletal muscle interleukin-6 regulates hepatic cytochrome P450 expression: effects of 16-Week high-fat diet and exercise. Toxicol Sci 2018;162:309-17.

32 Bertholdt L, Gudiksen A, Schwartz CL, et al. Lack of skeletal muscle IL-6 influences hepatic glucose metabolism in mice during prolonged exercise. Am J Physiol Regul Integr Comp Physiol 2017;312:R626-36.

33 Zhang $\mathrm{H}-\mathrm{J}, \mathrm{He}$ J, Pan L-L, et al. Effects of moderate and vigorous exercise on nonalcoholic fatty liver disease: a randomized clinical trial. JAMA Intern Med 2016;176:1074-82.
34 Wolf Greenstein A, Majumdar N, Yang P, et al. Hepatocyte-Specific, PPAR $\gamma$-regulated mechanisms to promote steatosis in adult mice. J Endocrinol 2017;232:107-21.

35 Matsusue K, Haluzik M, Lambert G, et al. Liver-Specific disruption of PPARgamma in leptin-deficient mice improves fatty liver but aggravates diabetic phenotypes. J Clin Invest 2003;111:737-47.

36 Brandt $\mathrm{C}$, Jakobsen $\mathrm{AH}$, Adser $\mathrm{H}$, et al. II-6 regulates exercise and training-induced adaptations in subcutaneous adipose tissue in mice. Acta Physiol 2012;205:224-35

37 McKie GL, Medak KD, Knuth CM, et al. Housing temperature affects the acute and chronic metabolic adaptations to exercise in mice. $J$ Physiol 2019;597:4581-600.

38 Raun SH, Henriquez-Olguín C, Karavaeva I, et al. Housing temperature influences exercise training adaptations in mice. Nat Commun 2020;11:1560. 